\title{
Editorial
}

\section{Diabetic vascular disease and the rising star of Protein Kinase C}

I have noticed in the pubs of Ireland there is little talk of protein kinase C (PKC). To readers of Diabetologia this may come as a great surprise: a glance at the Internet list will show that more than 6000 articles have appeared over the past 4 years, testifying to the explosion of interest in the kinase which phosphorylates various enzymes and many other cellular proteins and is also activated by second messengers in many biological systems. The controversy and confusion that surrounded PKC in the early days was a result of our ignorance. How were we to know that the original PKC was part of a large hidden tribe? And with the discovery of each new member of that PKC tribe, we are in a better position to explore their function in the scheme of things. For diabetologists these are exciting times because discoveries are being made which appear to throw light on a range of metabolic dysfunction found in diabetes, particularly in the vasculature and in the area of insulin release and action. The purpose of this short editorial is to encourage the reader to explore PKC in depth by turning to page (659-673) and reading the focussed and enlightening review by Idris et al. [1].

The PKC family is derived from multiple genes. Activation and translocation of many PKCs occurs in response to short-term increases in diacylglycerols (DAGs). Activation leads to a variety of biological responses such as cell proliferation and differentiation, glucose and lipid metabolism, smooth muscle contraction and gene expression. It has become clear that the family PKC might have a very important role to play in the tissue destruction found in diabetes. Furthermore, most PKCs are up regulated in diabetes. This is because one of the major metabolic disturbances is a release of non-esterified fatty acids and a rise in DAG. It is exciting to learn that specific fatty acids of varying chain length appear to activate PKC synergistically with DAG and in diabetes increased non-esterified fatty acid concentration could stimu- late PKC activity independently of de novo synthesis of DAG. That non-esterified fatty acids could be the primary defect in diabetes comes more into focus when one considers that higher concentrations of DAG could be the key biochemical link, by PKC activation, between abnormal lipid metabolism and insulin resistance. However, the discovery of new members of the PKC family confirms that the isoforms serve widely different functions and in a tissue-dependent manner. Thus some isoforms function as mediators of insulin action affecting both the insulin receptor and glucose transport. It is also interesting that another isoform could regulate the second phase insulin secretory response. These isoforms are not activated by DAG but appear to be stimulated by polyphosphoinositides derived from PI-kinase activation by insulin.

PKC is of interest not only because of its role in glucose homeostasis but also in the pathological events that cause diabetes complications. Increases in DAG occur in various tissues in diabetes. The endothelium has come to be seen as a very active organ and endothelial dysfunction as an early marker of vascular disease. Increased PKC activation seems to be an important biochemical modification in diabetes-related endothelial dysfunction and increased release of endothelin-1 in the retina, for example, is associated with PKC-mediated inhibition of constitutive nitric oxide synthase and reduced NO mediated vasodilatation. It has also been shown that PKC inhibition restores microvascular haemodynamics. Again it becomes clear that the various isoforms of PKC could increase or decrease NO production according to cell type, tissue location and duration of diabetes [2]. A pathway to endothelium release of nitric oxide is through the platelet release of platelet derived ADP. In diabetes this is impaired and is associated with an increase in DAG concentrations and PKCmediated stimulation of phospholipase A2 activity. 
PKC activity in monocytes has been shown to enhance their adhesion to the vascular wall and to promote their differentiation into macrophages, crucial to the development of atherosclerosis. Finally PKC is involved in the intercellular adhesion molecule-1 (ICAM-1) up regulation. Thus further understanding of the isoforms can be expected to lead to a much better understanding of both small and large vessel diabetic angiopathy and to provide a very good basis for the development of specifically targeted inhibitors or stimulants of the various PKC isoforms.

$\mathrm{PKC}$ and the glomerulus in diabetes is developing into a tremendously exciting story. The early changes in the glomerulus in the diabetic kidney appear to be PKC-related through their effect on fibronectin and type 4 collagen formation by the mesangial cells. High glucose also seems to stimulate mesangial cell proliferation through the $\mathrm{PKC} / \mathrm{NF} \varkappa \mathrm{B}$ pathway [3] and is also responsible for ICAM-1 increase thus unravelling to a certain extent the complex pathways involved in early diabetic nephropathy and perhaps explaining the mechanism of action of angiotensin converting inhibitors which reduce the glomerular PKC activity.

Tomlinson [4], at the end of 1999 , very successfully reviewed the evidence to support the role of $\mathrm{PKC}$ isoforms as a trigger for all the cellular events necessary for the development of diabetic complications and suggested that their pharmacological modulation might provide therapeutic control. The present review article in this journal strongly promotes the search for isozyme-selective PKC inhibitors and presents new and exciting evidence.

I will return to my local pub full of confidence in the knowledge that I can now interest my non-medical drinking friends in the excitement of the PKC story.

Prof. Gerald H. Tomkin,

The Adelaide and Meath Hospital,

1 Fitzwilliam Square, Dublin 2, Ireland

\section{References}

1. Idris I, Gray S, Donnelly R. (2001) Protein kinase C activation isoenzyme-specific effects on metabolic and cardiovascular function. Diabetologia 44: 659-673

2. Hopfiner RL, Gopolakrishnan V (1999) Endothelin Emerging roll in diabetic vascular complications. Diabetologia 42: 1383-1394

3. Park CW, Kim JH, Lee JW et al. (2000) High glucose intercellular adhesion molecule 1 (ICAM-1) expression through an osmotic effect in rat mesangial cells is PKC-NF- $x \mathrm{~B}-\mathrm{de}-$ pendent. Diabetologia 43: 1544-1553

4. Tomlinson DR (1999) Mitogen-activated protein kinases as glucose transducers for diabetic complications. Diabetologia 42: 1271-1281 